\title{
IL-10 and TGF- $B 1$ Cytokines Stimulated by Ascaris lumbricoides Body Fluid Are Associated with Secretory IgA Levels Against Other Intestinal Pathogens Attenuating Diarrheal Symptoms
}

\author{
Isabel Hagel ${ }^{1}\left[\right.$ ] Laura Díaz $^{1} \cdot$ Dennis Alexander Lugo $^{2} \cdot$ Maira Cabrera $^{2} \cdot$ María Cristina Di Prisco $^{3}$
}

Received: 17 May 2021 / Accepted: 19 July 2021 / Published online: 11 August 2021

(c) Dr. K C Chaudhuri Foundation 2021

To the Editor: It has been extensively reported that helminthderived excretory/secretory products stimulate, nonspecifically, the production of TGF $\beta$ and IL-10 regulatory cytokines by Foxp $3^{+} \mathrm{T}$ regulatory cells, generating a tolerant microenvironment in the gut, which allows them to persist for long periods of time preventing parasite-associated pathologies and modulating bystander inflammatory processes [1]. Thereby, the possible influence of Ascaris lumbricoides body fluid (ABF)-stimulated $\mathrm{IL}-10$ and TGF- $\beta$ cytokines on secretory IgA levels and diarrheal symptoms were studied in a group of Venezuelan slum preschool children. Clinical, parasitological, and bacteriological studies were carried out. sIgA levels in the sublingual/submandibular saliva fluids and cytokines in supernatants of ABF-stimulated whole blood cultures were measured by ELISA. Data were analyzed by nonparametric ANOVA. It was found that $69.2 \%$ of the children were helminth-parasitized with A. lumbricoides being the most prevalent parasite. By the time of examination, $32.7 \%$ had diarrheal symptoms mainly caused by Giardia duodenalis and Escherichia coli EPEC. Helminthic infection did not prevent from infections by other intestinal parasites. However, regardless of the infective pathogen, diarrheal symptoms were lower $(p<0.0001)$ among helminth-parasitized children. Values of ABF-stimulated TGF $\beta 1$ and IL-10 were higher $(p<0.005)$ among helminth parasitized children and correlated positively $(p<0.001)$ with total, anti-G. duodenalis and anti-E. coli EPEC sIgA values. Diarrhea symptoms were lower $(p<0.001)$ among children with elevated levels of $\operatorname{sgA}$. The results of this work

Isabel Hagel

Isabelhagel@gmail.com

1 Laboratory of Immunochemistry, Institute of Biomedicine, Faculty of Medicine, Central University of Venezuela, Caracas 1010 A, Venezuela

2 Laboratory of Immunoparasitology, Institute of Biomedicine, Faculty of Medicine, Central University of Venezuela, Caracas, Venezuela

3 Laboratory of Immunopathology, Faculty of Medicine, Central University of Venezuela, Caracas, Venezuela suggest that ABF-stimulated IL-10 and TGF- $\beta$ may enhance nonspecifically the production of total and specific sIgA against other enteric pathogens. Through this mechanism helminths may favor the development of anti-inflammatory mechanisms driven by secretory $\operatorname{IgA}$ [2]. Besides, the stimulation of high levels of specific sIgA against E. coli EPEC and G. duodenalis would enhance prevention of mucosal adhesion of these pathogens [3, 4], thus limiting inflammation and ameliorating intestinal symptoms. Nevertheless, further studies are needed to confirm these preliminary results.

Funding This work was financed by the Council for Scientific and Humanistic Development (CDCH) from the Central University of Venezuela (PROYECT No: PG-O9-8680-2013-1) and the Special Help Fund from the Research Directorate of the Faculty of Medicine, Central University of Venezuela.

\section{Declarations}

Conflict of Interest None.

\section{References}

1. Titz T de O, de Araújo CAA, Enobe CS, Rigato PO, Oshiro TM, de Macedo-Soares MF. Ascaris suum infection modulates inflammation: Implication of CD $4{ }^{+} \mathrm{CD} 25^{\text {high }}$ Foxp ${ }^{+} \mathrm{T}$ cells and IL-10. Parasite Immunol. 2017;39:e12453.

2. Mikulic J, Longet S, Favre L, Benyacoub J, Corthesy B. Secretory $\mathrm{IgA}$ in complex with Lactobacillus rhamnosus potentiates mucosal dendritic cell-mediated Treg cell differentiation via TLR regulatory proteins, RALDH 2 and secretion of IL- 10 and TGF- $\beta$. Cell Mol Immunol. 2017;14:546-56.

3. Cravioto A, Tello A, Villafan H, Ruiz J, Del Vedovo S, Neeser JR. Inhibition of localized adhesion of enteropathogenic Escherichia coli to HEp-2 cells by immunoglobulin and oligosaccharide fractions of human colostrum and breast milk. J Infect Dis. 1991;163:1247-55.

4. Jenkins MC, O'Brien CN, Murphy C, et al. Antibodies to the ventral disc protein $\delta$-giardin prevent in vitro binding of giardia lamblia trophozoites. J Parasitol. 2009;95:895-9.

Publisher's Note Springer Nature remains neutral with regard to jurisdictional claims in published maps and institutional affiliations. 